\title{
On topological properties of hierarchical hypercube network based on Ve and Ev degree
}

https://doi.org/10.1515/mgmc-2021-0022

received March 08, 2021; accepted May 12, 2021

\begin{abstract}
Grid implementation is a principal unit in electrical and electronic engineering but it depends on the domain of these projects. For example, depending on the grid and the signal processing in that fields of electronic and electrical engineering, such as more abstract mathematics in signal conversion and e-transmission theory griding, etc. Provides transmission through grid nodes. Graph theory is very useful in research fields. As topological indices, there are more actual numbers associated with chemical composition complaints connected to the chemical grid with physical and chemical properties and reactions. In this paper, we expand the work to interconnected grid and examine the first Zagreb, the second Zagreb, Randic, sum-connectivity, harmonic, geometric, and atom bond connectivity exponents of hierarchical hypercube network based on vertex-edge and edge-vertex degree.
\end{abstract}

Keywords: hierarchical hypercube network (HHN), edge-vertex degree, vertex-edge degree, molecular topological indices

\section{Introduction}

It usually requires a multi-processor interconnect grid to connect to thousands of analogs, copies of processor-memory pairs, each this pairing is called processing node. No need to use shared memory, all integration and transmission with processing nodes to execution of the program is usually completed by message transferring. Utilization and structure of

\footnotetext{
* Corresponding author: Nida Zahra and Muhammad Ibrahim, Centre for Advanced Studies in Pure and Applied Mathematics, Bahauddin Zakariya University Multan, Pakistan, e-mail: zahranida512@gmail.com; mibtufail@gmail.com
}

various processor linkage grid is recently captured more attention due to accessibility of cheap, solid nano processor and bit cache. Web grid has been known as universal interconnection grid as a large-scale linkage parallel computing. Web/ring like low dimensional grid, they have received more consideration recently due to their improvement, compared to more complex grid, it can be extended to larger grid, such as hypercube. Especially failure has studied reliance on network collaboration (Gutman et al., 2012), which have a lot lately. Algorithm development on optical system and extremal weights of graph are studied by Al-Ayyoub et al. (2006) and Bollobas and Erdos (1998). Basic operations (Wang and Sahni, 1998a), image processing (Wang and Sahni, 2000), and many more about the OTISMesh optoelectronic computer are studied by Wang and Sahni (1998b). To lessen the grid and to use the locus of transmission to lessen link costs exist in similar utilization, Hierarchical interconnection network (HIN) provide design framework. Various level are engaged by HIN. The core grid facilitates native transmission, whereas developed grids provides distant transmission. Various leveled grid have been in use for a long time. The transmission grid performs similar calculations (Konstantinidou, 1992). Some web networking topology characteristics are studied in Aslam et al. (2017), Baig and Naeem (2018), Hayat and Imran (2014), and Shao et al. (2018). Chemicals composite and their structure are designed by molecular diagrams. A molecular design is a design of atoms as points of the given structure and links are their linkage. Topological indices is a digital figure related to the structure describe the topological feature. It is invariant under the automorphism of the graph. More briefly, the topological descriptor, the $\operatorname{Top}(G)$ of graph $G$ is a number, and each graph $H$ that is isomorphic to graph $G$, $\operatorname{Top}(H)=\operatorname{Top}(G)$. The work completed by Wiener (1947) gave the idea of topological descriptor, while working at the breaking point of the paraffin. He called this indices the routing number. Then, the routing number was recalled as Wiener indices. This topological descriptor had provided base 
to the topological indices, from the perspective of theory and application, for details, see Dobrynin et al. (2001), Estrada et al. (1998), and Gutman and Polansky (1986). So chemically and quantitatively literature, the most commonly used topological lesion are Wiener, Zagreb, Randic, and atom-bond connectivity (Chen et al. 2012; Das et al. 2011; Ediz 2017a, 2017b; Li et al., 2010; Wiener, 1947; Zhou and Trinajstiae 2010; Zhou et al., 2013). In any in this case, all the above work has been completed using the previous degree concepts. Later on, Chellali et al. (2017) discovered two new degree ideas in grid history, specifically, vertex-edge degree, and edgevertex degree. Many researchers explore different networks by using these two new ideas such as Ediz (2018). These ideas are the transformation of previous degree-based concept (Estrada et al. 1998; Sahin and Ediz, 2018).

\section{Preliminaries}

This part comprises of some primary concepts related to graph which is usually represented by $H=(V, E)$ where $V$ represents collection of nodes and $E$ is collection of links of graph. The total number of links attached to a node is degree of that node. In connected graph there exist adjacency of nodes. The open neighborhood, denoted by $N(y)$, for a node $y$ can be defined as the $N(y)=x \in V \mid x, y \in E$. Similarly, the closed neighborhood of $y$, denoted by $N[y]$, can be defined as If we add the node $y$ to the collection of $N(y)$, then we get the edgevertex degree of the link $f=x y \in E$, denoted by $\tilde{\phi}_{e v}(f)$ defined in Cancan (2019) is equal to the number of nodes in the union of the closed neighborhoods of $x$ and $y$. Furthermore, The vertex-edge degree, denoted by $\tilde{\phi}_{v e}(y)$, defined in Cancan (2019) of the node $y \in V$, is equal to the number of links that are joined to any nodes from the closed neighborhood of $y$ but in this collection there is not repetition occur.

Let $H$ be a simple graph and $f=x y \in E(H)$. For detail associated to vertex-edge and edge-vertex degree topological indices, we mention (Cancan, 2019; Chellali et al., 2017).

In the given all descriptions, consider $H$ be a simple graph and $y \in V(H)$.

The edge-vertex degree Zagreb indices is defined as:

$$
\mathcal{M}^{\mathrm{ev}}(\mathrm{H})=\sum_{\mathrm{f} \in \mathrm{E}(\mathrm{H})} \tilde{\phi}_{\mathrm{ev}}(\mathrm{f})^{2}
$$

The first vertex-edge degree Zagreb alpha indices is define as:

$$
\mathcal{M}_{1}^{\text {ove }}(H)=\sum_{\chi \in V(G)} \tilde{\phi}_{v e}(x)^{2}
$$

The first vertex-edge degree Zagreb beta indices is define as:

$$
\mathcal{M}_{1}^{\beta v e}(H)=\sum_{x y \in E(H)}\left(\tilde{\phi}_{v e}(x)+\tilde{\phi}_{v e}(y)\right)
$$

The second vertex-edge degree Zagreb indices is define as:

$$
\mathcal{M}_{2}^{v e}(H)=\sum_{x y \in E(H)}\left(\tilde{\phi}_{v e}(x) \times \tilde{\phi}_{v e}(y)\right)
$$

The vertex-edge degree Randic indices is define as:

$$
\mathcal{R}^{v e}(H)=\sum_{x y \in E(H)}\left(\tilde{\phi}_{v e}(x) \times \tilde{\phi}_{v e}(y)\right)^{-\frac{1}{2}}
$$

The edge-vertex degree Randic indices is define as:

$$
\mathcal{R}^{e v}(H)=\sum_{f \in E(H)} \tilde{\phi}_{v e}(f)^{-\frac{1}{2}}
$$

The vertex-edge degree atom-bond connectivity indices is define as:

$$
\mathcal{A B C}^{v e}(H)=\sum_{x y \in E(H)} \sqrt{\frac{\tilde{\phi}_{v e}(x)+\tilde{\phi}_{v e}(y)-2}{\tilde{\phi}_{v e}(x) \times \tilde{\phi}_{v e}(y)}}
$$

The vertex-edge degree geometric-arithmetic (ve-GA) indices is define as:

$$
\mathcal{G A}^{v e}(H)=\sum_{x y \in E(H)} \frac{2 \sqrt{\tilde{\phi}_{v e}(x) \times \tilde{\phi}_{v e}(y)}}{\tilde{\phi}_{v e}(x)+\tilde{\phi}_{v e}(y)}
$$

The vertex-edge degree harmonic (ve-H) indices is defined as:

$$
\mathcal{H}^{v e}(H)=\sum_{x y \in E(H)} \frac{2}{\tilde{\phi}_{v e}(x)+\tilde{\phi}_{v e}(y)}
$$

The vertex-edge degree sum-connectivity (ve- $\chi$ ) indices is defined as:

$$
\chi^{v e}(H)=\sum_{x y \in E(H)}\left(\tilde{\phi}_{v e}(x)+\tilde{\phi}_{v e}(y)\right)^{-\frac{1}{2}}
$$




\section{Hierarchical hypercube network $(H H N-1)_{n \times n}$}

In this segment, we determine some vertex-edge and edge-vertex degree based topological indices for hierarchical hypercube network $(H H N-1)$, from Guirao et al. (2018), see Figure 1. Let $(H H N-1)_{n \times n}$ be a hierarchical hypercube network. The $(H H N-1)_{n \times n}$ have $16 n+16$ nodes and $24 n+20$ links. We divide the link collection with respect to the degrees of end nodes of each link of $(H H N-1)_{n \times n}$ into two component. One link component $E_{1}\left((H H N-1)_{n \times n}\right)$ comprises of 16 links $u v$, where $\operatorname{deg}(u)=2, \operatorname{deg}(v)=3$. The other link part $E_{2}\left((H H N-1)_{n \times n}\right)$ comprises of $24 n+4$ links $u v$, where $\operatorname{deg}(u)=\operatorname{deg}(v)=3$.

Similarly, we divide the node collection with respect to the degrees of nodes into two components. One component comprises the 8 nodes with degree 2 and the other is comprises the $16 n+8$ nodes with degree 3.

We splits the links, based on edge-vertex degree of the $(H H N-1)_{n \times n}$ for $n \geq 2$ in Table 1. Similarly, we splits the nodes and links, based on vertex-edge degree of $(H H N-1)_{n \times n}$ for $n \geq 2$ in Tables 2 and 3.

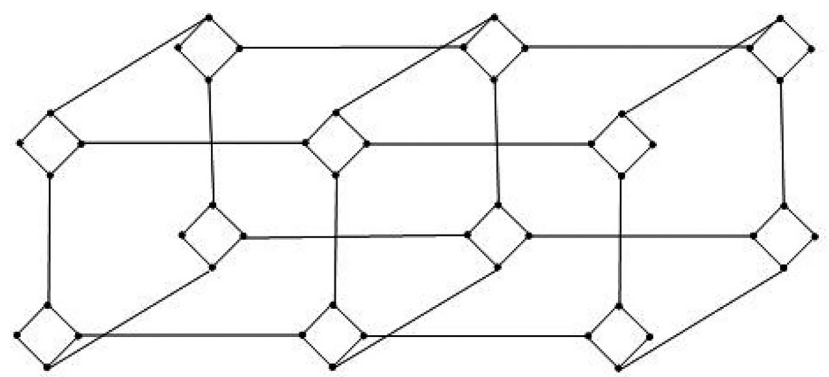

Figure 1: Hierarchical hypercube network $(\mathrm{HNN}-1)_{2 \times 2}$.

Table 1: Link partitioning of $(H H N-1)_{n \times n}$

\begin{tabular}{lcc}
\hline No. of links & Deg of end nodes & Edge-vertex degrees \\
\hline 16 & $(2,3)$ & 5 \\
$24 n+4$ & $(3,3)$ & 6 \\
\hline
\end{tabular}

Table 2: Nodes partitioning of $(H H N-1)_{n \times n}$

\begin{tabular}{lcc}
\hline No. of nodes & Deg(u) & Vertex-edge degrees \\
\hline 8 & 2 & 6 \\
16 & 3 & 8 \\
$16 n-8$ & 3 & 9 \\
\hline
\end{tabular}

Table 3: The vertex-edge degree of the end nodes of links of $(H H N-1)_{n \times n}$

\begin{tabular}{lcc}
\hline No. of links & Deg of end nodes & $\begin{array}{c}\text { Vertex-edge degrees } \\
\text { of end nodes }\end{array}$ \\
\hline 16 & $(2,3)$ & $(6,8)$ \\
16 & $(3,3)$ & $(8,9)$ \\
$24 n-12$ & $(3,3)$ & $(9,9)$ \\
\hline
\end{tabular}

\subsection{Edge-vertex degree base indices}

Now for the hierarchical hypercube network $(H H N-1)_{n \times n}$, we will determine Zagreb and Randic indices based on the edge-vertex degree.

\subsubsection{Zagreb index}

The Zagreb index using Table 1 is computed as:

$$
\begin{aligned}
& \mathcal{M}^{e v}(H H N-1)=\sum_{f \in E(H H N-1)} \tilde{\phi}_{e v}(f)^{2}, \\
& \mathcal{M}^{e v}(H H N-1)=16 \times 5^{2}+(24 n+4) \times 6^{2} \\
& =400+864 n+144 \\
& =864 n+544 .
\end{aligned}
$$

\subsubsection{The Randic index}

The Randic index using Table 1 is computed as:

$$
\begin{aligned}
& \mathcal{R}^{e v}(H H N-1)=\sum_{f \in E(H H N-1)} \tilde{\phi}_{e v}(f)^{-\frac{1}{2}}, \\
& \mathcal{R}^{e v}(H H N-1)=16 \times 5^{-\frac{1}{2}}+(24 n+4) \times 6^{-\frac{1}{2}} \\
& =\frac{16}{\sqrt{5}}+\frac{24}{\sqrt{6}} n+\frac{4}{\sqrt{6}} \\
& =9.79 n+8.78 .
\end{aligned}
$$

\subsection{Vertex-edge degree base indices}

Now for the hierarchical hypercube network $(H H N-1)_{n \times n}$, we will determine the vertex-edge degree based first Zagreb alpha, first Zagreb beta, the second Zagreb, Randic, atombond connectivity, geometric-arithmetic, harmonic, and sum-connectivity indices. 


\subsubsection{The first Zagreb alpha index}

The first Zagreb alpha index using Table 2 can be computed as:

$$
\begin{aligned}
& \mathcal{M}_{1}^{\text {ove }}(H H N-1)=\sum_{x \in V(H H N-1)} \tilde{\phi}_{v e}(x)^{2}, \\
& \mathcal{M}_{1}^{\text {ove }}(H H N-1)=8 \times 6^{2}+16 \times 8^{2}+(16 n-8) \times 9^{2} \\
& =288+1024+1296 n-648 \\
& =1296 n+664 .
\end{aligned}
$$

\subsubsection{The first Zagreb beta index}

The first Zagreb beta index using Table 3 can be computed as:

$$
\begin{aligned}
& \mathcal{M}_{1}^{\beta v e}(H H N-1)=\sum_{x y \in E(H H N-1)}\left(\tilde{\phi}_{v e}(x)+\tilde{\phi}_{v e}(y)\right), \\
& \mathcal{M}_{1}^{\beta v e}(H H N-1)=16 \times 14+16 \times 17+(24 n-12) \times 18 \\
& =224+272+432 n-216 \\
& =432 n+280 .
\end{aligned}
$$

\subsubsection{The second Zagreb index}

The second Zagreb index using Table 3 can be computed as:

$$
\begin{aligned}
& \mathcal{M}_{2}^{v e}(H H N-1)=\sum_{x y \in E(H H N-1)}\left(\tilde{\phi}_{v e}(x) \times \tilde{\phi}_{v e}(y)\right) \\
& \mathcal{M}_{2}^{v e}(H H N-1)=16 \times 48+16 \times 72+(24 n-12) \times 81 \\
& =768+1152+1944 n-972 \\
& =1944 n+948 .
\end{aligned}
$$

\subsubsection{The Randic index}

The Randic index using Table 3 can be computed as:

$$
\begin{aligned}
& \mathcal{R}^{v e}(H H N-1)=\sum_{x y \in E(H H N-1)}\left(\tilde{\phi}_{v e}(x) \times \tilde{\phi}_{v e}(y)\right)^{-\frac{1}{2}}, \\
& \mathcal{R}^{v e}(H H N-1)=16 \times 48^{-\frac{1}{2}}+16 \times 72^{-\frac{1}{2}}+(24 n-12) \times 81^{-\frac{1}{2}}
\end{aligned}
$$

$$
\begin{aligned}
& =\frac{4}{\sqrt{3}}+\frac{8}{3 \sqrt{2}}+\frac{8}{3} n-\frac{4}{3} \\
& =2.67 n+2.86 .
\end{aligned}
$$

\subsubsection{The atom-bond connectivity index}

The atom-bond connectivity index using Table 3 can be computed as:

$$
\begin{aligned}
& \mathcal{A B C}^{v e}(H H N-1)=\sum_{x y \in E(H H N-1)} \sqrt{\frac{\tilde{\phi}_{v e}(x)+\tilde{\phi}_{v e}(y)-2}{\tilde{\phi}_{v e}(x) \times \tilde{\phi}_{v e}(y)}}, \\
& \mathcal{A B C}^{v e}(H H N-1)=16 \times \sqrt{\frac{14-2}{48}}+16 \times \sqrt{\frac{17-2}{72}}
\end{aligned}
$$

$$
\begin{aligned}
& +(24 n-12) \times \sqrt{\frac{18-2}{81}} \\
& =8+\frac{8 \sqrt{15}}{\sqrt{2}}+\frac{32}{3} n-\frac{16}{3} \\
& =10.67 n+24.57 .
\end{aligned}
$$

\subsubsection{The geometric-arithmetic index}

The geometric arithmetic index using Table 3 can be computed as:

$$
\begin{aligned}
& \mathcal{G} \mathcal{A}^{v e}(H H N-1)=\sum_{x y \in E(H H N-1)} \frac{2 \sqrt{\tilde{\phi}_{v e}(x) \times \tilde{\phi}_{v e}(y)}}{\tilde{\phi}_{v e}(x)+\tilde{\phi}_{v e}(y)}, \\
& \mathcal{G} \mathcal{A}^{v e}(H H N-1)=16 \times \frac{2 \sqrt{48}}{14}+16 \times \frac{2 \sqrt{72}}{17}+(24 n-12) \times \frac{2 \sqrt{81}}{18} \\
& =\frac{64 \sqrt{3}}{7}+\frac{192 \sqrt{2}}{17}+24 n-12 \\
& =24 n+19.81 .
\end{aligned}
$$

\subsubsection{The harmonic index}

The harmonic index using Table 3 can be computed as:

$$
\mathcal{H}^{v e}(H H N-1)=\sum_{x y \in E(H H N-1)} \frac{2}{\tilde{\phi}_{v e}(x)+\tilde{\phi}_{v e}(y)},
$$




$$
\begin{aligned}
& \mathcal{H}^{\text {ve }}(H H N-1)=16 \times \frac{2}{14}+16 \times \frac{2}{17}+(24 n-12) \times \frac{2}{18} \\
& =\frac{16}{7}+\frac{32}{17}+\frac{8}{3} n-\frac{4}{3} \\
& =2.67 n+2.83 .
\end{aligned}
$$

\subsubsection{The sum-connectivity index}

The sum-connectivity index using Table 3 can be computed as:

$$
\begin{aligned}
& \chi^{v e}(H H N-1)=\sum_{x y \in E(H H N-1)}\left(\tilde{\phi}_{v e}(x)+\tilde{\phi}_{v e}(y)\right)^{-\frac{1}{2}}, \\
& \chi^{v e}(H H N-1)=16 \times 14^{-\frac{1}{2}}+16 \times 17^{-\frac{1}{2}}+(24 n-12) \times 18^{-\frac{1}{2}} \\
& =\frac{16}{\sqrt{14}}+\frac{16}{\sqrt{17}}+\frac{8}{\sqrt{2}} n-\frac{4}{\sqrt{2}} \\
& =5.66 n+5.33 .
\end{aligned}
$$

\section{Hierarchical hypercube network $(H H N-2)_{n \times n}$}

In this segment, we determine some vertex-edge and edgevertex degree based topological indices for hierarchical hypercube network (HHN-2), from Guirao et al. (2018), see in Figure 2. The $(H H N-2)_{n \times n}$ have $16 n+16$ nodes and $32 n+28$ links. We divide the link collection with respect to the degrees of end nodes of each link of $(H H N-2)_{n \times n}$ into two components. One link component $E_{1}\left((H H N-2)_{n \times n}\right)$ comprises of 24 links $u v$, where $\operatorname{deg}(u)=3, \operatorname{deg}(v)=4$. The other link component $E_{2}\left((H H N-2)_{n \times n}\right)$ comprises of $32 n+4$ links $u v$, where $\operatorname{deg}(u)=\operatorname{deg}(v)=4$.

Similarly, we divide the node collection with respect to the degrees of nodes into two components. One

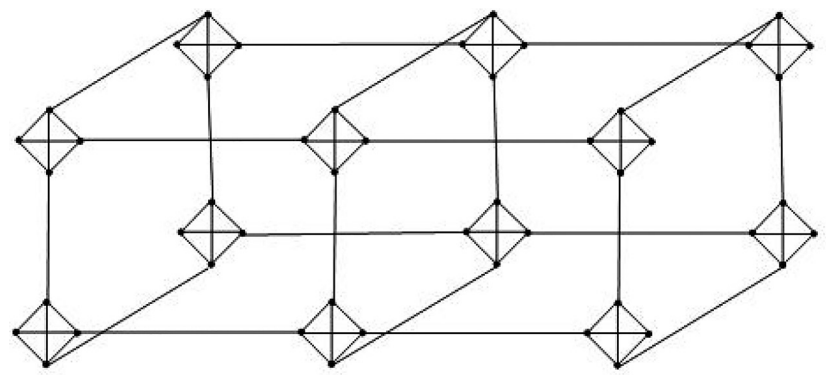

Figure 2: Hierarchical hypercube network $(\mathrm{HNN}-2)_{2 \times 2}$ component comprises of 8 nodes with degree 3 and the other component comprises of $16 n+8$ nodes with degree 4 .

We split the links, based on edge-vertex degree of the $(H H N-2)_{n \times n}$ for $n \geq 2$ in Table 4. Similarly, we divide the nodes and links, based on vertex-edge degree of $(H H N-2)_{n \times n}$ for $n \geq 2$ in Tables 5 and 6 .

\subsection{Edge-vertex degree base indices}

Now for the hierarchical hypercube network $(H H N-1)_{n \times n}$ we will compute Zagreb and Randic indices based on the edge-vertex degree.

\subsubsection{Zagreb index}

The Zagreb index using Table 4 can be computed as:

$$
\begin{aligned}
& \mathcal{M}^{e v}(H H N-2)=\sum_{e \in E(H H N-1)} \tilde{\phi}_{e v}(e)^{2}, \\
& \mathcal{M}^{e v}(H H N-2)=24 \times 5^{2}+24 n \times 6^{2}+(8 n+4) \times 8^{2} \\
& =600+864 n+512 n+256 \\
& =1376 n+856 .
\end{aligned}
$$

Table 4: Link partitioning of $(H H N-2)_{n \times n}$

\begin{tabular}{lcc}
\hline No. of links & Deg of end nodes & Edge-vertex degrees \\
\hline 24 & $(3,4)$ & 5 \\
$24 n$ & $(4,4)$ & 6 \\
$8 n+4$ & $(4,4)$ & 8 \\
\hline
\end{tabular}

Table 5: Nodes partitioning of $(H H N-2)_{n \times n}$

\begin{tabular}{lcc}
\hline No. of nodes & Deg(u) & Vertex-edge degrees \\
\hline 8 & 3 & 9 \\
24 & 4 & 12 \\
$16 n-16$ & 4 & 13 \\
\hline
\end{tabular}

Table 6: The vertex-edge degree of the end nodes of links of $(H H N-2)_{n \times n}$.

\begin{tabular}{lcc}
\hline No. of links & Deg of end nodes & $\begin{array}{c}\text { Vertex-edge degrees } \\
\text { of end nodes }\end{array}$ \\
\hline 24 & $(3,4)$ & $(9,12)$ \\
32 & $(4,4)$ & $(12,12)$ \\
8 & $(4,4)$ & $(12,13)$ \\
$32 n-36$ & $(4,4)$ & $(13,13)$ \\
\hline
\end{tabular}




\subsubsection{The Randic index}

The Randic index using Table 4 can be computed as:

$$
\begin{aligned}
& \mathcal{R}^{e v}(H H N-2)=\sum_{e \in E(H H N-1)} \tilde{\phi}_{e v}(e)^{-\frac{1}{2}}, \\
& \mathcal{R}^{e v}(H H N-2)=24 \times 5^{-\frac{1}{2}}+24 n \times 6^{-\frac{1}{2}}+(8 n+4) \times 8^{-\frac{1}{2}} \\
& =\frac{24}{\sqrt{5}}+\frac{24}{\sqrt{6}} n+\frac{4}{\sqrt{2}} n+\frac{2}{\sqrt{2}} \\
& =12.63 n+12.15 .
\end{aligned}
$$

\subsection{Vertex-edge degree base indices}

Now for the hierarchical hypercube network $(H H N-1)_{n \times n}$, we will compute first Zagreb alpha, first Zagreb beta, the second Zagreb, Randic, atom-bond connectivity, geometric-arithmetic, harmonic, and sum-connectivity indices based on the vertex-edge degree.

\subsubsection{The first Zagreb alpha index}

The first Zagreb alpha index using Table 5 can be computed as:

$$
\begin{aligned}
& \mathcal{M}_{1}^{\text {ove }}(H H N-1)=\sum_{v \in V(H H N-1)} \tilde{\phi}_{v e}(v)^{2}, \\
& \mathcal{M}_{1}^{\text {ove }}(H H N-1)=8 \times 9^{2}+24 \times 12^{2}+(16 n-16) \times 13^{2} \\
& =648+3456+2704 n-2704 \\
& =2704 n+1400 .
\end{aligned}
$$

\subsubsection{The first Zagreb beta index}

The first Zagreb beta index using Table 6 can be computed as:

$$
\begin{aligned}
& \mathcal{M}_{1}^{\beta v e}(H H N-2)=\sum_{u v \in E(H H N-1)}\left(\tilde{\phi}_{v e}(u)+\tilde{\phi}_{v e}(v)\right), \\
& \mathcal{M}_{1}^{\beta v e}(H H N-2)=24 \times 21+32 \times 24+8 \times 25+(32 n-36) \times 26 \\
& =504+768+200+832 n-936 \\
& =832 n+536 .
\end{aligned}
$$

\subsubsection{The second Zagreb index}

The second Zagreb index using Table 6 can be computed as:

$$
\begin{aligned}
& \mathcal{M}_{2}^{v e}(H H N-2)=\sum_{u v \in E(H H N-1)}\left(\tilde{\phi}_{v e}(u) \times \tilde{\phi}_{v e}(v)\right), \\
& \mathcal{M}_{2}^{v e}(H H N-2)=24 \times 108+32 \times 144+8 \times 156 \\
& +(32 n-36) \times 169 \\
& =2592+4608+1248+5408 n-6084 \\
& =5408 n+2364 .
\end{aligned}
$$

\subsubsection{The Randic index}

The Randic index using Table 6 can be computed as:

$$
\begin{aligned}
\mathcal{R}^{v e}(H H N-2) & =\sum_{u v \in E(H H N-1)}\left(\tilde{\phi}_{v e}(u) \times \tilde{\phi}_{v e}(v)\right)^{-\frac{1}{2}}, \\
\mathcal{R}^{v e}(H H N-2) & =24 \times 108^{-\frac{1}{2}}+32 \times 144^{-\frac{1}{2}}+8 \times 156^{-\frac{1}{2}} \\
& +(32 n-36) \times 169^{-\frac{1}{2}} \\
& =\frac{4}{\sqrt{3}}+\frac{8}{3}+\frac{4}{\sqrt{39}}+\frac{32}{13} n-\frac{36}{13} \\
& =2.46 n+2.85 .
\end{aligned}
$$

\subsubsection{The atom-bond connectivity index}

The atom-bond connectivity index using Table 6 can be computed as:

$$
\begin{aligned}
& \mathcal{A B C}^{v e}(H H N-2)=\sum_{u v \in E(H H N-1)} \sqrt{\frac{\tilde{\phi}_{v e}(u)+\tilde{\phi}_{v e}(v)-2}{\tilde{\phi}_{v e}(u) \times \tilde{\phi}_{v e}(v)}}, \\
& \mathcal{A B C}^{v e}(H H N-2)=24 \times \sqrt{\frac{21-2}{108}}+32 \times \sqrt{\frac{24-2}{144}}+8
\end{aligned}
$$

$$
\begin{aligned}
& \times \sqrt{\frac{25-2}{156}}+(32 n-36) \times \sqrt{\frac{26-2}{169}} \\
= & \frac{4 \sqrt{19}}{\sqrt{3}}+\frac{8 \sqrt{22}}{3}+\frac{4 \sqrt{23}}{\sqrt{39}}+\frac{64 \sqrt{6}}{13} n-\frac{72 \sqrt{6}}{13} \\
= & 12.06 n+12.08 .
\end{aligned}
$$




\subsubsection{The geometric-arithmetic index}

The geometric arithmetic index using Table 6 can be computed as:

$$
\begin{aligned}
\mathcal{G A}^{v e}(H H N-2) & =\sum_{u v \in E(H H N-1)} \frac{2 \sqrt{\tilde{\phi}_{v e}(u) \times \tilde{\phi}_{v e}(v)}}{\tilde{\phi}_{v e}(u)+\tilde{\phi}_{v e}(v)}, \\
\mathcal{G A}^{v e}(H H N-2) & =24 \times \frac{2 \sqrt{108}}{21}+32 \times \frac{2 \sqrt{144}}{24}+8 \times \frac{2 \sqrt{156}}{25} \\
& +(32 n-36) \times \frac{2 \sqrt{169}}{26} \\
& =\frac{96 \sqrt{3}}{7}+32+\frac{32 \sqrt{39}}{25}+32 n-36 \\
& =32 n+27.75 .
\end{aligned}
$$

\subsubsection{The harmonic index}

The harmonic index using Table 6 can be computed as:

$$
\begin{aligned}
& \mathcal{H}^{v e}(H H N-2)=\sum_{u v \in E(H H N-1)} \frac{2}{\tilde{\phi}_{v e}(u)+\tilde{\phi}_{v e}(v)}, \\
& \mathcal{H}^{v e}(H H N-2)=24 \times \frac{2}{21}+32 \times \frac{2}{24}+8 \times \frac{2}{25}+(32 n-36) \times \frac{2}{26} \\
& =\frac{16}{7}+\frac{8}{3}+\frac{16}{25}+\frac{32}{13} n-\frac{36}{13} \\
& =2.46 n+2.82 .
\end{aligned}
$$

\subsubsection{The sum-connectivity index}

The sum-connectivity index using Table 6 can be computed as:

$$
\begin{aligned}
& \chi^{v e}(H H N-2)=\sum_{u v \in E(H H N-1)}\left(\tilde{\phi}_{v e}(u)+\tilde{\phi}_{v e}(v)\right)^{-\frac{1}{2}}, \\
& \chi^{v e}(H H N-2)=24 \times 21^{-\frac{1}{2}}+32 \times 24^{-\frac{1}{2}}+8 \times 25^{-\frac{1}{2}} \\
& \quad+(32 n-36) \times 26^{-\frac{1}{2}} \\
& =\frac{24}{\sqrt{21}}+\frac{16}{\sqrt{6}}+\frac{8}{5}+\frac{32}{\sqrt{26}} n-\frac{36}{\sqrt{26}} \\
& =6.27 n+6.31 .
\end{aligned}
$$

\section{Graphical representation and discussion}

In this part, we debate diagrammatically illustration relative to the edge-vertex degree and vertex-edge degree based topological indices for the hierarchical hypercube network $(H H N-1)$ and $(H H N-2)$. We manipulate the precise results for the edge-vertex degree based Zagreb and Randic indices, vertex-edge degree based First Zagreb alpha, first Zagreb beta, the second Zagreb, Randic, atombond connectivity (ve- $\mathcal{A B C}$ ), geometric-arithmetic (ve- $\mathcal{G A})$, harmonic (ve- $\mathcal{H}$ ), and sum-connectivity (ve- $\chi$ ) indices for the hierarchical hypercube network $H H N-1$ and $H H N-2$.

The diagrammatically illustration of hierarchical hypercube network $H H N-1$ are given below. All indicators grow with growing values of $n$, can be noticed from given Figures 3 and 4. It can also be detected that vertex-edge

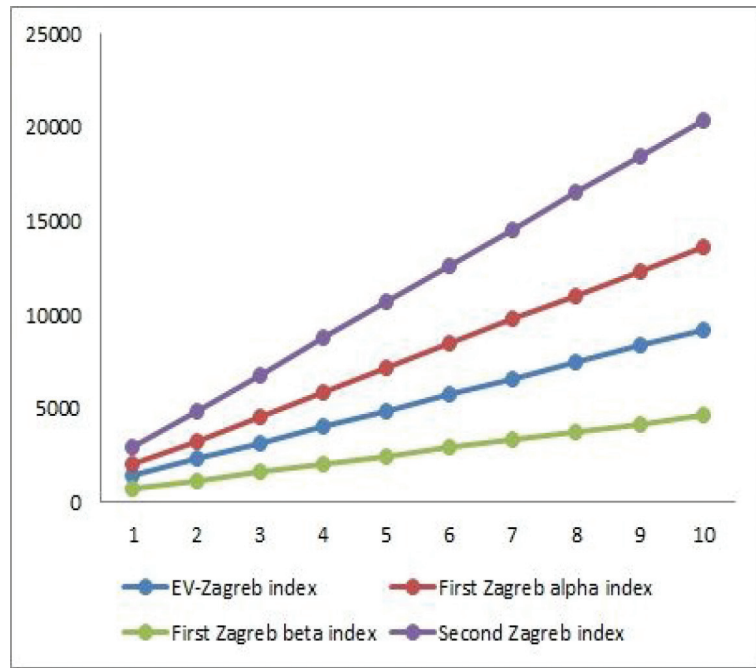

Figure 3: Graphical comparison of $M^{e v}, M_{1}^{o v e}, M_{1}^{\beta v e}$, and $M_{2}^{v e}$ for HNN-1.

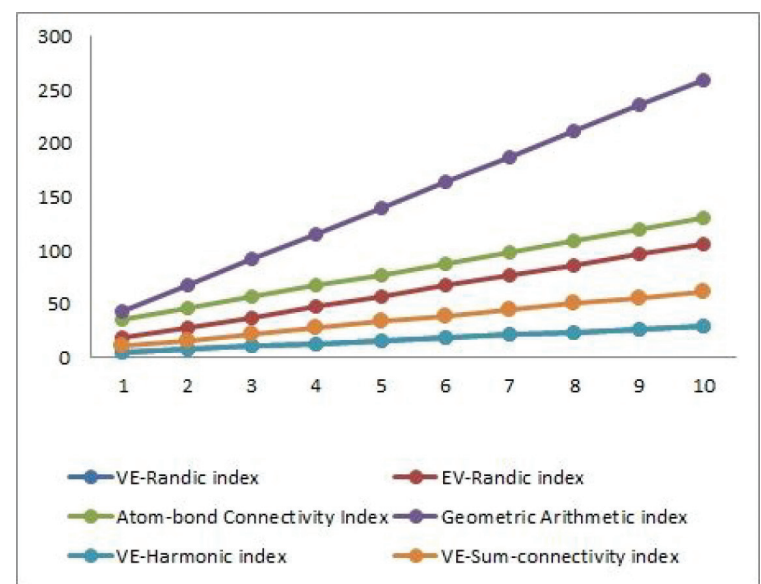

Figure 4: Graphical comparison of $R^{e v}, G A^{v e}, A B C^{v e} H^{v e}, R^{v e}$, and $\chi^{v e}$ for HNN-1. 
degree based Randic and harmonic indices behave same for all values of $n$.

Similarly, The diagrammatically illustration of hierarchical hypercube network $H H N-2$ are given below. All indicators expand with expansion of $n$, can be noticed from given Figures 5 and 6. It can also be noticed that vertex-edge degree based Randic and harmonic indices act same for all values of $n$.

\section{Conclusion}

Research the network through graphs, topological indices are important to understand its fundamental topology. Such researches has a global utilization in the computer field science, which uses various indices based on graph invariance to deal with some provocation scenarios. In the investigation of quantitative structure-activity

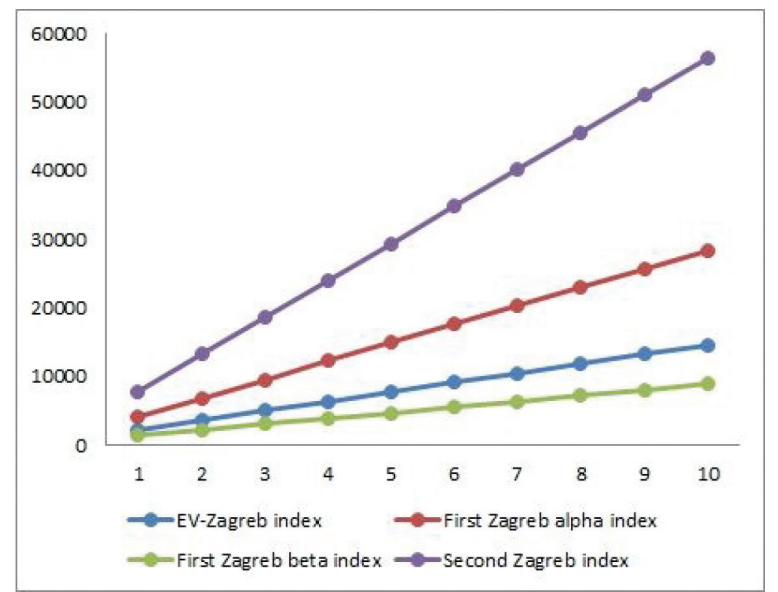

Figure 5: Graphical comparison of $M^{e v}, M_{1}^{o v e}, M_{1}^{\beta v e}$, and $M_{2}^{v e}$ for HNN-2.

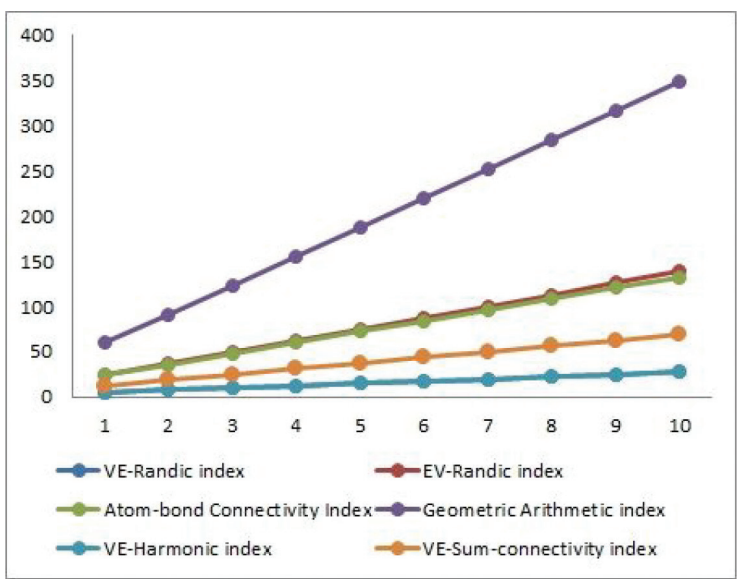

Figure 6: Graphical comparison of $R^{e v}, G A^{v e}, A B C^{v e} H^{v e}, R^{v e}$, and $\chi^{v e}$ for HNN-2. relationship (QSAR) and quantitative structure-property relationship (QSPR), figure invariants are important tools for approximation and predicateization the characteristics of chemical networking.

In this paper, we have presented some outcomes with respect to the edge-vertex degree and vertex-edge degree based indices such as the edge-vertex degree Zagreb and Randic indices, vertex-edge degree based First Zagreb alpha, first Zagreb beta, the second Zagreb, Randic, atombond connectivity (ve- $\mathcal{A B C}$ ), geometric-arithmetic (ve- $\mathcal{G A}$ ), harmonic (ve- $\mathcal{H}$ ), and sum-connectivity (ve- $\chi$ ) indices for the hierarchical hypercube network $H H N-1$ and $H H N-2$.

Acknowledgement: Authors are grateful to anonymous referees for nice suggestion that improved the final version of the paper.

Funding information: Authors state no funding involved.

Author contribution: All authors contribute equally.

Conflict of interest: Authors state no conflict of interest.

\section{References}

Al-Ayyoub A., Awwad A., Day K., Ould-Khaoua M., Generalized methods for algorithm development on optical systems. J. Supercomput., 2006, 38, 111-125.

Aslam A., Guirao J.L.G., Ahmad S., Gao W., Topological indices of the line graph of subdivision graph of complete bipartite graphs. Appl. Math. Inf. Sci., 2017, 11, 1631-1636.

Baig A.Q., Naeem M., Revan and hyper revan indices of octahedral and icosahedral networks. App. Math. Nonl. Sci., 2018, 3, 33-40.

Bollobas B., Erdos P., Graphs of extremal weights. Ars Combinatoria, 1998, 50, 225-233.

Cancan M., On edge-vertex degree and vertex-edge degree Topological Properties of Tickysim Spiking Neural Network. Comput. Intel. Neurosc., 2019, 8, 11-31.

Chellali M., Haynes T.W., Hedetniemi S.T., Lewis T.M., On ve-degrees and ev-degrees in graphs. Discrete Math., 2017, 340(2), 31-38.

Chen J., Liu J., Guo X., Some upper bounds for the atom-bond connectivity indices of graphs. Appl. Math. Lett., 2012, 25, 1077-1081.

Das K.C., Gutman I., Furtula B., On atom-bond connectivity indices. Chem. Phys. Lett., 2011, 511, 452-454.

Dobrynin A.A., Entringer R., Gutman I., Wiener index of trees: Theory and applications. Acta Appl. Math., 2001, 66, 211-249.

Ediz S., Predicting some Physicochemical Properties of Octane Isomers. A Topological Approach Using ev-Degree and ve-Degree Zagreb Indices. Int. J. System Sci. Appl. Math., 2017a, 9(4), 87-94.

Ediz S., A New Tool for QSPR Researchers: ev-Degree Randic Index. Celal Bayar Univ. J. Sci., 2017b, 13(3), 615-618. 
Ediz S., On ve-degree Molecular Topological Properties of Silicate and Oxygen Network. Int. J. System Sci. Appl. Math., 2018, 9(1), 1-12.

Estrada E., Torres L., Rodriguez L., Gutman I., An atom-bond connectivity indices: Modelling the enthalpy of formation of alkanes. Indian J. Chem., 1998, 37, 849-855.

Guirao J.L.G., Siddiqui M.K., Hussain A., On topological properties of block shift and hierarchical hypercube networks. Open Phys., 2018, 16, 810-819.

Gutman I., Polansky O.E., Mathematical Concepts in Organic Chemistry. Springer-Verlag, New York, 1986.

Gutman I., Tošoviæ J., Radenkovi S., Markovi S., On atom-bond connectivity index and its chemical applicability. Indian J. Chem., 2012, 51A, 690-694.

Hayat S., Imran M., Computation of topological indices of certain networks. Appl. Math. Comput., 2014, 240, 213-228.

Konstantinidou S., The selective extra stage butterfly. IEEE T. VLSI Syst., 1992, 1, 502-506.

Li Y., Li F., Zhou Z., Chen Z., SiC 2 silagraphene and its one-dimensional derivatives: where planar tetracoordinate silicon happens. J. Am. Chem. Soc., 2010, 133(4), 900-908.
Sahin B., Ediz S., On ev-Degree and ve-Degree Topological Indices. Iran. J. Math. Chem., 2018, 9(4), 236-277

Shao Z., Siddiqui M.K., Muhammad M.H., Computing Zagreb Indices and Zagreb Polynomials for Symmetrical Nanotubes. Symmetry, 2018, 10, 1-20.

Wang C.F., Sahni S., Basic operations on the OTIS-Mesh optoelectronic computer. IEEE T. Parall. Distr., 1998a, 9, 1226-1236.

Wang C.F., Sahni S., Image processing on the OTIS-Mesh optoelectronic computer. IEEE T. Parall. Distr., 2000, 11, 97-109.

Wang C.F., Sahni S., Chapter 5: OTIS optoelectronic computers. In: Li K., Pan Y., Zheng S.-Q. (Eds.), Parallel Computation Using Optical Interconnection. Kluwer Academic Publishers, 1998b, 99-116.

Wiener H., Structural determination of parafln boiling points. J. Am. Chem. Soc., 1947, 69, 17-20.

Zhou B., Trinajstiæ N., On general sum-connectivity indices. J. Math. Chem., 2010, 47, 210-218.

Zhou L.J., Zhang Y.F., Wu L.M., $\mathrm{SiC}_{2}$ siligraphene and nanotubes: novel donor materials in excitonic solar cells. Nano Lett., 2013, 13(11), 5431-5436. 